\title{
Stability analysis of a simple walking model driven by an oscillator with a phase reset using sensory feedback
}

AUTHOR(S):

Aoi, S; Tsuchiya, $\mathrm{K}$

\section{CITATION:}

Aoi, S ... [et al]. Stability analysis of a simple walking model driven by an oscillator with a phase reset using sensory feedback. IEEE TRANSACTIONS ON ROBOTICS 2006, 22(2): 391397

\section{ISSUE DATE:}

2006-04

URL:

http://hdl.handle.net/2433/50545

\section{RIGHT:}

(c)2006 IEEE. Personal use of this material is permitted. However, permission to reprint/republish this material for advertising or promotional purposes or for creating new collective works for resale or redistribution to servers or lists, or to reuse any copyrighted component of this work in other works must be obtained from the IEEE. 


\title{
Stability Analysis of a Simple Walking Model Driven by an Oscillator With a Phase Reset Using Sensory Feedback
}

Shinya Aoi and Kazuo Tsuchiya

\begin{abstract}
This paper deals with the analytical examination of the dynamic properties of the walking motion of a biped robot based on a simple model. The robot is driven by rhythmic signals from an oscillator, which receives feedback signals from touch sensors at the tips of the legs. Instantly, the oscillator resets its phase and modifies the walking motion according to the feedback signals. Based on such a simple model, approximate periodic solutions are obtained, and the stability of the walking motion is analytically investigated by using a Poincaré map. The analytical results demonstrate that the modification of the step period and the walking motion due to the sensory feedback signals improves the stability of the walking motion.
\end{abstract}

Index Terms-Central pattern generator (CPG), phase reset, Poincaré map, sensory feedback signal, stability analysis.

\section{INTRODUCTION}

Rhythmic motions such as animal walking are achieved by interaction between the dynamics of a musculoskeletal system and the rhythmic signals from the central pattern generator (CPG) [6], [12]. The CPG comprises a set of neural oscillators present in the spinal cord, and spontaneously generates rhythmic signals even if it does not receive outer signals, such as a sensor signal. However, it is very sensitive to outer signals and modifies the rhythmic signals influenced by the outer signals, resulting in adaptive motions. CPG is widely modeled using nonlinear oscillators. Many studies have been carried out in order to elucidate the role for CPG in locomotion using quadruped robots [4], [16], biped robots [1], [10], [15], a simulated salamander [8], and human models [11], [13], [14], [17]. In particular, a recent approach that incorporates the resetting of the CPG's phase, depending on outer signals, has been proposed [1], [10], [15]-[17]. For example, in our previous work [1], [15], a locomotion control system for a biped robot was developed using nonlinear oscillators. The nominal trajectories of the joints are designed by maps from the phases of the oscillators. A controller composed of nonlinear oscillators receives feedback signals from touch sensors. Instantly, the phase of the oscillator is reset, and the nominal trajectories of the joints are modified according to the phase reset. Studies revealed that the phase reset achieves robust walking motions by numerical simulations and hardware experiments. However, they are not sufficient to clarify the adaptability mechanism. The purpose of this paper is to clarify

Manuscript received February 7, 2005; revised June 7, 2005. This paper was recommended for publication by Associate Editor Q. Huang and Editor H. Arai upon evaluation of the reviewers' comments. This work was supported in part by the Center of Excellence for Research and Education on Complex Functional Mechanical Systems (COE Program of the Ministry of Education, Culture, Sports, Science and Technology, Japan), and in part by a Grant-in-Aid for Scientific Research on Priority Areas "Emergence of Adaptive Motor Function through Interaction between Body, Brain, and Environment" from the Japanese Ministry of Education, Culture, Sports, Science and Technology. This paper was presented at the International Conference on Systems, Man, and Cybernetics, Hague, The Netherlands, October 2004.

The authors are with the Department of Aeronautics and Astronautics, Graduate School of Engineering, Kyoto University, Kyoto 606-8501, Japan (e-mail: shinya_aoi@kuaero.kyoto-u.ac.jp; tsuchiya@kuaero.kyoto-u.ac.jp).

Digital Object Identifier 10.1109/TRO.2006.870671 


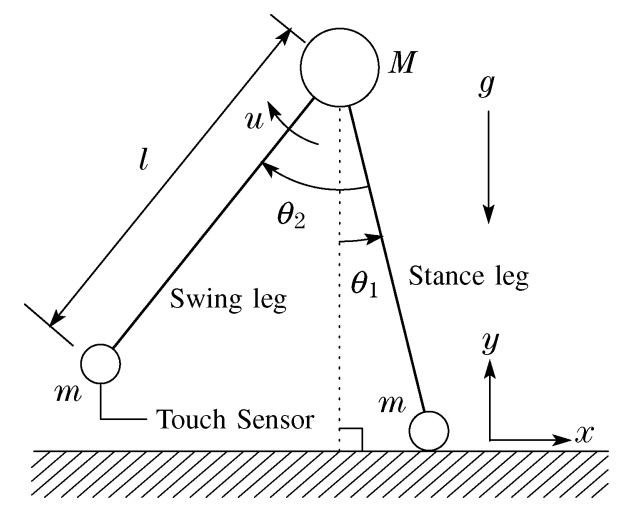

Fig. 1. Schematic model of a simple walking model of a biped robot.

the role for the phase reset in adaptive walk based on a simple biped walking model.

\section{SimPLE WALKING MODEL}

\section{A. Biped Robot Model}

Fig. 1 shows a simple walking model of a biped robot composed of a hip and two legs, a swing leg and a stance leg. The legs are connected at the hip and the leg length is $l$. A touch sensor is attached to the tip of each leg. The tip of the stance leg is constrained on the ground, and the stance leg can only rotate around the tip. It is assumed that hip mass $M$ and leg mass $m$ are concentrated at the hip and at the tip of the leg, respectively, and that the robot is constrained on the $x-y$ plane where the walking direction is the $x$ axis. This system has two degrees of freedom, $\theta_{1}$ and $\theta_{2}$, where $\theta_{1}$ is the angle of the stance leg with respect to the perpendicular line to the ground and $\theta_{2}$ is the angle between the swing leg and the stance leg. The robot has an oscillator that drives the robot whose amplitude is $\gamma$ and whose phase is $\phi$. Acceleration due to gravity is $g$.

The step cycle of the robot consists of two types of successive phases, a single-supported phase and a double-supported phase. In the singlesupported phase, only the stance leg is in contact with the ground, while in the double-supported phase, both legs are in contact with the ground. In the single-supported phase, since the robot has no actuator between the stance leg and the ground, the stance leg is not controlled directly. On the other hand, the robot has an actuator to manipulate the swing leg with respect to the stance leg. That is, angle $\theta_{1}$ is not controlled directly, while angle $\theta_{2}$ is controlled by actuator torque $u$.

\section{B. Single-Supported Phase}

In the single-supported phase, dimensionless equations of motion are given by

$$
\begin{aligned}
& {\left[\begin{array}{cc}
1+2 \beta\left(1-c \theta_{2}\right) & -\beta\left(1-c \theta_{2}\right) \\
-\left(1-c \theta_{2}\right) & 1
\end{array}\right]\left[\begin{array}{l}
\ddot{\theta}_{1} \\
\ddot{\theta}_{2}
\end{array}\right]+} \\
& {\left[\begin{array}{c}
-\beta \mathrm{s} \theta_{2}\left(\dot{\theta}_{2}^{2}-2 \dot{\theta}_{1} \dot{\theta}_{2}\right) \\
-\dot{\theta}_{1}^{2} \mathrm{~s} \theta_{2}
\end{array}\right]+} \\
& {\left[\begin{array}{c}
\beta \mathrm{s}\left(\theta_{1}-\theta_{2}\right)-\beta \mathrm{s} \theta_{1}-\mathrm{s} \theta_{1} \\
-\mathrm{s}\left(\theta_{1}-\theta_{2}\right)
\end{array}\right]=\left[\begin{array}{c}
0 \\
u^{\prime}
\end{array}\right]}
\end{aligned}
$$

where $c x \equiv \cos x, \mathrm{~s} x \equiv \sin x, \beta \equiv m / M>0, u^{\prime} \equiv u / m g l, \tau \equiv$ $\sqrt{g / l} t, t$ indicates time, and, from now on, $\dot{*}$ indicates the derivative of variable $*$ with respect to $\tau$.

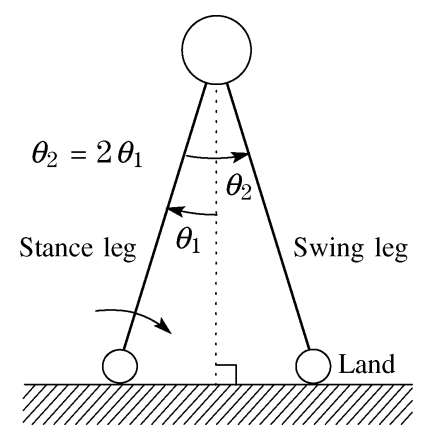

Fig. 2. Geometric condition for double-supported phase.

Also, in the single-supported phase, amplitude $\gamma$ of the oscillator is constant and phase $\phi$ of the oscillator has constant angular velocity $\omega(>0)$, i.e.,

$$
\dot{\gamma}=0 \quad \dot{\phi}=\omega
$$

Actuator torque $u^{\prime}$ manipulates angle $\theta_{2}$ using a feedback control. Here, desired angle $\theta_{2 d}$ of controlled angle $\theta_{2}$ is designed so that the robot is driven by a rhythmic signal from the oscillator. Since the motion of the swing leg can be modeled as a pendulum-like oscillation [5], [9], desired angle $\theta_{2 d}$ is designed as the simple function of amplitude $\gamma$ and phase $\phi$ of the oscillator and constant parameter $\eta$ by

$$
\theta_{2 d}=\theta_{2 d}(\phi, \gamma, \eta)=\gamma c \phi+\eta
$$

A condition is imposed on desired angle $\theta_{2 d}$ to take value $-S$ at $\phi=\pi$ as stride angle. Then, it yields $\eta=\gamma-S$ and, thus, the desired angle $\theta_{2 d}$ is modified by

$$
\theta_{2 d}=\theta_{2 d}(\phi, \gamma)=\gamma c \phi+\gamma-S
$$

Using desired angle $\theta_{2 d}$, actuator torque $u^{\prime}$ is given by

$$
u^{\prime}=-K_{p}\left\{\theta_{2}-\theta_{2 d}(\gamma, \phi)\right\}-K_{d}\left\{\dot{\theta}_{2}-\dot{\theta}_{2 d}(\gamma, \phi)\right\}
$$

where $K_{p}$ and $K_{d}$ are gain constants.

\section{Double-Supported Phase}

When the swing leg lands on the ground, both of the legs are in contact with the ground, as shown in Fig. 2. The condition that brings the double-supported phase is called the double-support condition and is geometrically given by

$$
r(q) \equiv 2 \theta_{1}-\theta_{2}=0
$$

It is assumed that the double-supported phase duration is sufficiently short, which indicates that immediately following the double-supported phase, the swing leg is, in turn, constrained on the ground and the stance leg leaves the ground. That is, the foot constraint on the ground changes between the two legs, and the swing leg instantaneously becomes the stance leg and vice versa. This assumption implies that an impulsive force may occur at the tip of the swing leg and result in a discontinuous change in the angular velocities. It is also assumed that the stance leg lifts from the ground without interaction 


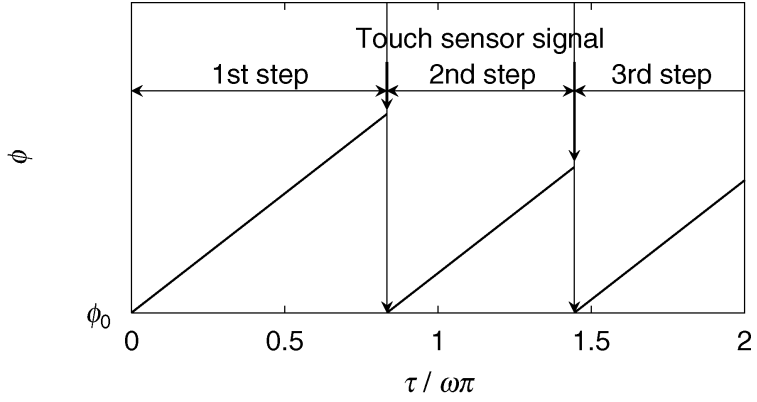

(a)

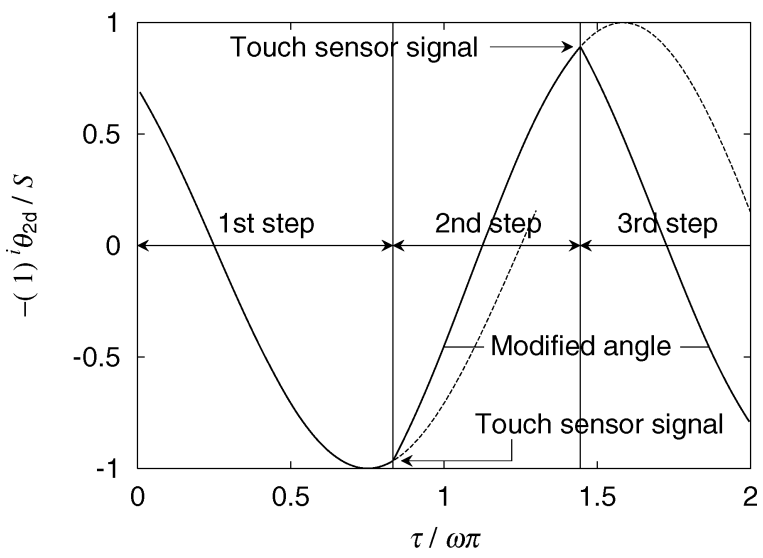

(b)

Fig. 3. Modification due to feedback signals from touch sensor. (a) and (b) show phase $\phi$ and desired angle $-(-1)^{i} \theta_{2 d} / S$, respectively, where $i$ is the step number. Phase $\phi$ is reset to $\phi_{0}$, and desired angle $\theta_{2 d}$ is modified to start the next step according to feedback signals from the touch sensor. (a) Phase reset. (b) Modification of $\theta_{2 d}$.

and that, during the short double-supported phase, the influence of the actuator torque is too small and can be ignored. From the conservation of the angular momentum and the condition of the swing leg to be constrained on the ground just after the double-supported phase, the relationship between the states immediately prior to and immediately following the double-supported phase is given by

$$
\left[\begin{array}{c}
\theta_{1}^{+} \\
\dot{\theta}_{1}^{+} \\
\theta_{2}^{+} \\
\dot{\theta}_{2}^{+}
\end{array}\right]=\left[\begin{array}{c}
-\theta_{1}^{-} \\
\frac{2 c 2 \theta_{1}^{-}}{2+\beta\left(1-c 4 \theta_{1}^{-}\right)} \dot{\theta}_{1}^{-} \\
-\theta_{2}^{-} \\
\frac{2 c 2 \theta_{1}^{-}\left(1-c 2 \theta_{1}^{-}\right)}{2+\beta\left(1-c 4 \theta_{1}^{-}\right)} \dot{\theta}_{1}^{-}
\end{array}\right]
$$

where $*^{-}$and $*^{+}$indicate the state immediately prior to and immediately following a double-supported phase, respectively (for example, see [7] for details).

When the swing leg touches the ground, the oscillator receives a feedback signal from the touch sensor. Instantly, the phase of the oscil- lator is reset to a certain value $\phi_{0}$, and then the walking motion is modified to start the next step according to the phase reset (see Fig. 3). In the modification, from (7), condition $\theta_{2 d}^{+}=-\theta_{2 d}^{-}$is imposed on desired angle $\theta_{2 d}$ not to change discontinuously between immediately prior to and immediately following double-supported phases. As a result, the amplitude of the oscillator is modified, and the relationship between the states for the oscillator just before and just after the double-supported phase is given by

$$
\left[\begin{array}{l}
\gamma^{+} \\
\phi^{+}
\end{array}\right]=\left[\frac{2 S-\gamma^{-}\left(1+c \phi^{-}\right)}{1+c \phi_{0}}\right]
$$

Relations (7) and (8) are called transition rules.

\section{LINEAR ANALYSIS}

In this paper, angle $\theta_{2}$ is controlled by a feedback control in (5). It is assumed that as long as angle $\theta_{2}$ is controlled by sufficient high-gain feedback control torque, angle $\theta_{2}$ can catch up with desired angle $\theta_{2 d}$ in a sufficiently small period of time, and thus angle $\theta_{2}$ is identical to desired angle $\theta_{2 d}$, i.e.,

$$
\theta_{2}(\tau)=\theta_{2 d}(\gamma(\tau), \phi(\tau))
$$

In light of the above description, state variables are defined as

$$
q^{T}=\left[\theta_{1} \dot{\theta}_{1} \gamma \phi\right]
$$

A set of equations is given from equations of motion (1), double-support condition (6), and transition rules (7) and (8) by

$$
\begin{cases}\dot{q}=f(q), & q^{-} \notin S_{\mathrm{c}} \\ q^{+}=h\left(q^{-}\right), & q^{-} \in S_{\mathrm{c}}\end{cases}
$$

where $S_{c}:=\{q \mid r(q)=0\}$ and the following definitions are given, along with the one shown at the bottom of the page:

$$
\begin{aligned}
f(q) \equiv\left[\begin{array}{c}
\dot{\theta}_{1} \\
f_{2}(q) \\
0 \\
\omega
\end{array}\right] \\
h(q) \equiv\left[\begin{array}{c}
-\theta_{1} \\
\frac{2 c 2 \theta_{1}}{2+\beta\left(1-c 4 \theta_{1}\right)} \dot{\theta}_{1} \\
\frac{2 S-\gamma(1+c \phi)}{1+c \phi_{0}} \\
\phi_{0}
\end{array}\right] .
\end{aligned}
$$

\section{A. Periodic Solutions}

In this paper, walking motion is considered, in which the tipping motion of the robot from the perpendicular line to the ground is small. In that case, angles $\theta_{1}$ and $\theta_{2 d}$ are small. Equations and conditions are linearized and, thus, the periodic solutions from just following a

$$
f_{2}(q)=\frac{\left\{\beta\left(1-\mathrm{c} \theta_{2 d}\right) \ddot{\theta}_{2 d}+\beta \mathrm{s} \theta_{2 d}\left(\dot{\theta}_{2 d}^{2}-2 \dot{\theta}_{1} \dot{\theta}_{2 d}\right)-\beta \mathrm{s}\left(\theta_{1}-\theta_{2 d}\right)+\beta \mathrm{s} \theta_{1}+\mathrm{s} \theta_{1}\right\}}{\left\{1+2 \beta\left(1-\mathrm{c} \theta_{2 d}\right)\right\}}
$$




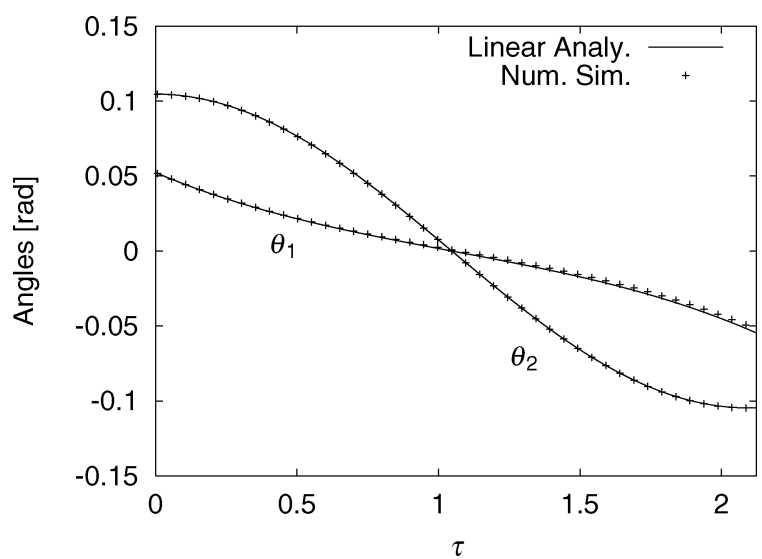

Fig. 4. Periodic solutions ( $\omega=1.5 \mathrm{rad}, \beta=0.3, S=6^{\circ}$, and $\left.\phi_{0}=0^{\circ}\right)$.

double-supported phase to the next double-supported phase are obtained in the following way. First, equations of motion (10) give

$$
\left\{\begin{array}{l}
\ddot{\theta}_{1}(\tau)-\theta_{1}(\tau)=\beta \theta_{2 d}(\gamma(\tau), \phi(\tau)) \\
\dot{\gamma}(\tau)=0 \\
\dot{\phi}(\tau)=\omega
\end{array} .\right.
$$

Double-support condition (6) and transition rules (10) become

$$
\left\{\begin{array}{l}
2 \theta_{1}(0)=\theta_{2 d}(\gamma(0), \phi(0)) \\
\theta_{1}(0)=-\theta_{1}(T) \\
\dot{\theta}_{1}(0)=\dot{\theta}_{1}(T) \\
\theta_{2 d}(\gamma(0), \phi(0))=-\theta_{2 d}(\gamma(T), \phi(T)) \\
\phi(0)=\phi_{0}
\end{array}\right.
$$

where $\tau=0$ and $\tau=T$ indicate the time immediately following a double-supported phase and the time immediately prior to the next double-supported phase, respectively. Solutions to (11) and (12) are obtained by

$$
\begin{aligned}
\theta_{1}(\tau)= & S c \phi_{0}\left(\frac{\beta}{\omega^{2}+1}+\frac{1}{2}\right)\left(\frac{e^{\tau}}{1-e^{T}}+\frac{e^{-\tau}}{1-e^{-T}}\right) \\
& -\frac{\beta S}{\omega^{2}+1} \mathrm{c}\left(\omega \tau+\phi_{0}\right), \quad 0 \leq \tau \leq T \\
\gamma(\tau)= & S, \quad 0 \leq \tau \leq T \\
\phi(\tau)= & \omega \tau+\phi_{0}, \quad 0 \leq \tau \leq T \\
T= & \frac{\pi-2 \phi_{0}}{\omega} .
\end{aligned}
$$

It follows that

$$
\theta_{2 d}(\gamma(\tau), \phi(\tau))=S c\left(\omega \tau+\phi_{0}\right), \quad 0 \leq \tau \leq T
$$

These solutions imply that the step period and the walking motion are modified depending on value $\phi_{0}$. Here, the results are shown using the following parameters: $\omega=1.5 \mathrm{rad}, \beta=0.3, S=6^{\circ}$, and $\phi_{0}=0^{\circ}$. Fig. 4 shows the periodic solutions obtained by linear analysis (Linear Analy.) and numerical simulation (Num. Sim.). Note that numerical simulation is based on the original nonlinear equations and conditions. Fig. 5 demonstrates the behavior of the state of the oscillator $\gamma(\tau) e^{j\{\phi(\tau)+i \pi\}}$, where $i(=0,1, \cdots)$ is the step number, by numerical simulation.

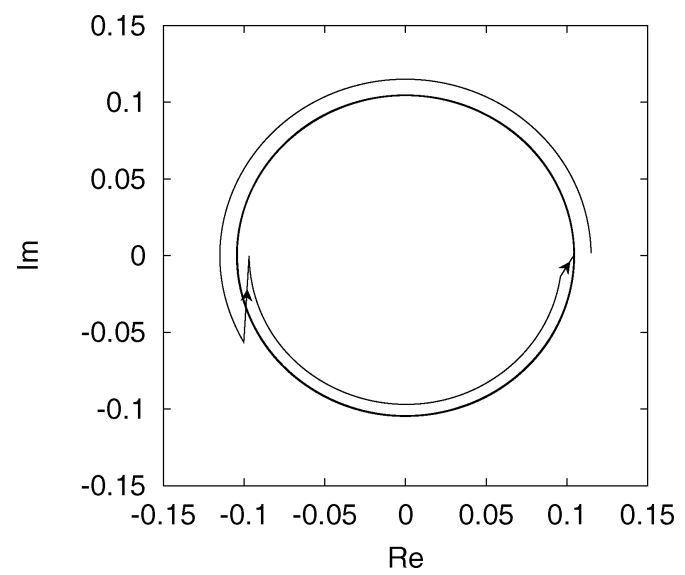

Fig. 5. Behavior of oscillator ( $\omega=1.5 \mathrm{rad}, \beta=0.3, S=6^{\circ}$, and $\left.\phi_{0}=0^{\circ}\right)$. Arrows indicate the phase reset due to touch sensor signals.

\section{STABILITY ANALYSIS}

\section{A. Poincaré Map}

In this section, the stability of the walking motion is analytically examined by using a Poincaré section. The state just after a double-supported phase is used as the state on the Poincaré section. The Poincaré map, which is the return map from one point on the Poincaré section to the next point on the Poincaré section, is denoted as $q \mapsto p(q)$, and then

$$
q_{i+1}^{+}=p\left(q_{i}^{+}\right)
$$

where $q_{i}^{+}$is the state immediately following the $i$ th double-supported phase. Note that fixed point $q^{*}$ on the Poincaré section satisfies

$$
q^{*}=p\left(q^{*}\right) .
$$

By adding perturbation $\hat{q}_{i}^{+}$from fixed point $q^{*}$ just after the $i$ th double-supported phase, where $\hat{*}$ indicates the perturbation, and linearizing Poincaré map $p$ at fixed point $q^{*}$, a Jacobian matrix $J\left(q^{*}\right)$ of the Poincaré map satisfies

$$
\hat{q}_{i+1}^{+}=J\left(q^{*}\right) \hat{q}_{i}^{+} .
$$

Periodic walk is asymptotically stable if all of the eigenvalues of Jacobian matrix $J\left(q^{*}\right)$ are inside the unit circle on the complex plane, that is, all of the magnitudes of the eigenvalues are less than 1.

\section{B. Jacobian Matrix of the Poincaré Map}

Since this system has a discontinuous change in the angular velocities between immediately before and immediately after a double-supported phase, the influences of the discontinuity need to be considered to find Jacobian matrix $J\left(q^{*}\right)$ of the Poincaré map at fixed point $q^{*}$. In [3], Coleman et al. show that Jacobian matrix $J\left(q^{*}\right)$ is equivalent to the product of three matrices $B, D$, and $E$ shown below. First, the periodic solution is defined as $q^{*}(\tau)$, the step period as $\tau^{*}$, and the perturbed state from periodic solution $q^{*}(\tau)$ from just after the $i$ th double-supported phase to the next double-supported phase as $q^{*}(\tau)+\hat{q}_{i}(\tau)$, where $\hat{q}_{i}(\tau)$ is subject to the initial condition $\hat{q}_{i}(0)=\hat{q}_{i}^{+}$. Then, matrices $B$ and $D$ are given by

$$
\begin{aligned}
& B=D_{q} h\left(q^{*}\left(\tau^{*}\right)\right) \\
& D=I-\frac{\dot{q}^{*}\left(\tau^{*}\right) D_{q} r\left(q^{*}\left(\tau^{*}\right)\right)^{\mathrm{T}}}{D_{q} r\left(q^{*}\left(\tau^{*}\right)\right)^{\mathrm{T}} \dot{q}^{*}\left(\tau^{*}\right)}
\end{aligned}
$$


where $I$ is a unit matrix and $D_{q} \equiv \partial / \partial q$. The evolved perturbation after the $i+1$ th double-supported phase $\hat{q}_{i+1}^{+}$satisfies

$$
\hat{q}_{i+1}^{+}=B D \hat{q}_{i}\left(\tau^{*}\right)
$$

The substitution of perturbed state $q^{*}(\tau)+\hat{q}_{i}(\tau)$ into the equations of motion (10) gives

$$
\dot{\hat{q}}_{i}(\tau)=D_{q} f\left(q^{*}(\tau)\right) \hat{q}_{i}(\tau)
$$

Matrix $E$ is derived by integrating (20) as follows:

$$
\hat{q}_{i}\left(\tau^{*}\right)=E \hat{q}_{i}^{+} .
$$

\section{Stability Region With Respect to Parameters}

In the previous section, periodic solutions (13) were obtained based on a simple walking model with feedback signals from the touch sensor. Here, the stability of the periodic solutions is investigated. The substitution of solutions (13) into (18) and (21) gives matrix $B D E$ [see (27), (29), and (31) in the Appendix]. Matrix $B D E$ has two zero eigenvalues $\left(\lambda_{3,4}=0\right)$, and the other two eigenvalues $\lambda_{1,2}$ are obtained from

$$
\lambda^{2}-2 a_{1} \lambda+a_{2}=0
$$

where

$$
\begin{aligned}
a_{1}= & \frac{1}{2 \dot{\theta}_{1}^{-}-\dot{\theta}_{2 d}^{-}} \\
& \times\left\{-\ddot{\theta}_{1}^{-} E_{-}+\dot{\theta}_{1}^{-}\left(E_{+}-\frac{1-c \phi_{0}}{1+c \phi_{0}}\right)+\frac{\dot{\theta}_{2 d}^{-}}{1+c \phi_{0}}\right. \\
& \times\left[\frac{\beta}{\omega^{2}+1}\left(\left(E_{+}+1\right) c \phi_{0}-E_{-} \omega s \phi_{0}\right)\right.
\end{aligned}
$$

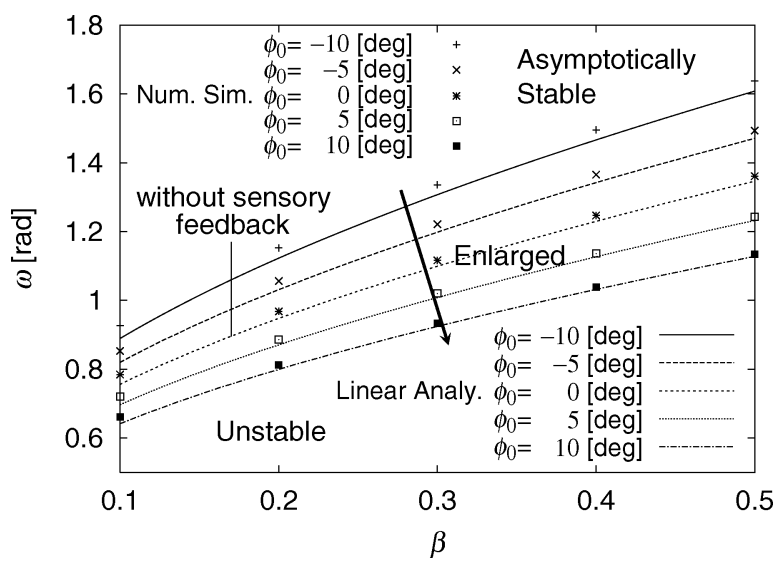

Fig. 6. Transition of stability region with respect to $\phi_{0}$.

Thus, eigenvalues $\lambda_{1,2}$ are obtained by the functions of parameters $\omega$, $\beta$, and $\phi_{0}$ and are given by

$$
\lambda_{1,2}\left(\omega, \beta, \phi_{0}\right)=a_{1} \pm \sqrt{a_{1}^{2}-a_{2}} .
$$

First, and particularly, the case when $\phi_{0}=0$ is considered. Then, (23) becomes equivalent to

$$
\lambda_{1,2}(\omega, \beta, 0)=1-\beta \omega^{2} d, 0
$$

where $d \equiv\left(\left(e^{\pi / \omega}+e^{-\pi / \omega}-2\right) /\left(1+2 \beta+\omega^{2}\right)\right)>0$. These eigenvalues reveal that the periodic solutions are asymptotically stable for $\beta \omega^{2} d<2$ and that the periodic solutions are unstable for $\beta \omega^{2} d>2$. With respect to the stability region of parameters $\beta$ and $\omega$, this result is the same as for the case in which the oscillator does not receive any feedback signals from external sensors, walking motion cannot be modified, and the robot is driven by open-loop rhythmic signals. However, by incorporating feedback signals, the stability is improved (see [2] for details). When the walking motion becomes unstable depending on parameters such as $\omega$ and $\beta$, consecutive period-doubling bifurcations occur, and the walking motion leads to chaotic motion (see [18]).

Next, the case that includes $\phi_{0} \neq 0$ is considered. Fig. 6 shows the transition of the stability region with respect to parameters $\beta$ and $\omega$, due to parameter $\phi_{0}$ and the comparison of the analytic and the numerical results. In the numerical simulations, parameter $S$ is set at $6^{\circ}$. This figure reveals that the asymptotic stability region is enlarged by increasing parameter $\phi_{0}$. However, when parameter $\phi_{0}$ exceeds a critical value which depends on parameters $\beta$ and $\omega$, the appearance of the stability region significantly changes. When parameter $\phi_{0}$ is less than the critical value, the decrease of $\beta$ or/and the increase of $\omega$ increases the stability of the periodic motion, and there is only one boundary with respect to parameters $\beta$ and $\omega$, which divides into asymptotically stable and unstable regions, as shown in Fig. 6. On the other hand, when parameter $\phi_{0}$ is beyond the critical value, another boundary appears and the asymptotically stable region is formed between two unstable regions with respect to parameters $\beta$ and $\omega$ (see Fig. 7). Therefore, in that case, there is a region where the decrease of $\beta$ or/and the increase of $\omega$ decreases the stability, and, thus, it is necessary to use an adequate value for parameter $\phi_{0}$. This paper focuses on the stability to achieve such a parameter $\phi_{0}$. Fig. 8(a) and (b) shows the maximum magnitude of the eigenvalues versus parameter $\phi_{0}$ with respect to parameters $\beta$ and $\omega$, respectively, revealing that it has an extreme value with respect to parameter $\phi_{0}$. Therefore, we use it as an optimal value of parameter $\phi_{0}$. Such an optimal value $\Phi_{0}$ is obtained by the condition $a_{1}^{2}-a_{2}=0$. 


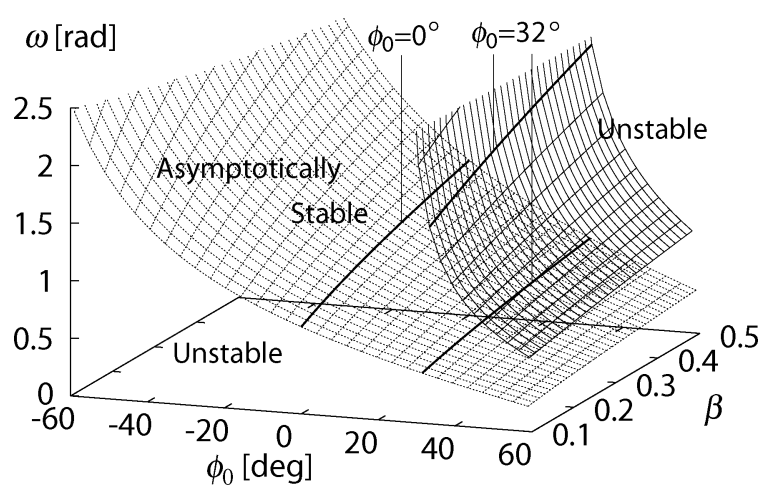

Fig. 7. Stability region with respect to the parameters.

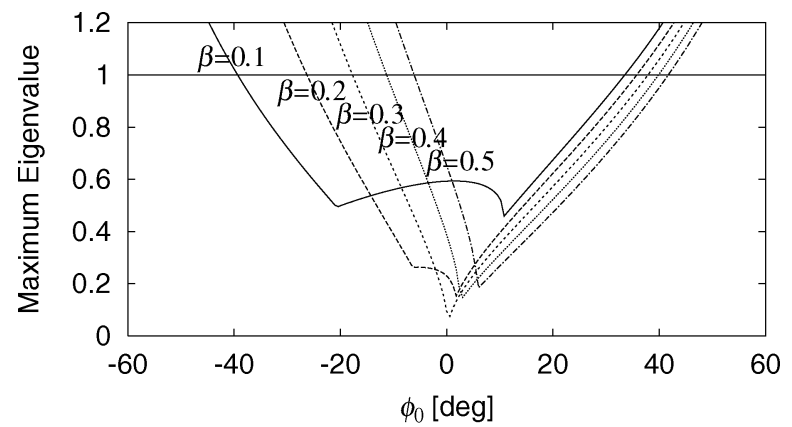

(a)

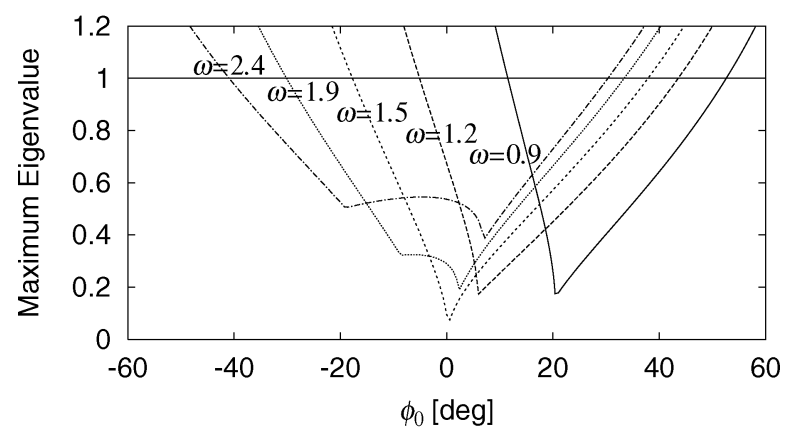

(b)

Fig. 8. Maximum magnitude of the eigenvalues with respect to $\phi_{0}$. (a) With respect to $\beta(\omega=1.5 \mathrm{rad})$. (b) With respect to $\omega \operatorname{rad}(\beta=0.3)$.

In particular, when optimal value $\Phi_{0}$ is small, from the linear analysis, it is given by

$$
\Phi_{0}=\frac{\left(1-\beta \omega^{2} d\right)^{2}}{\beta\left(1-\beta \omega^{2} d\right)\left\{\frac{16}{1+2 \beta+\omega^{2}}-\left(\omega^{2}-4\right) d\right\}-2} .
$$

Fig. 9 shows optimal value $\Phi_{0}$ with respect to parameters $\beta$ and $\omega$ obtained by the linear analysis and the rigorous numerical analysis. This analysis concludes that resetting the phase of the oscillator into an adequate value and modifying the walking motion due to the feedback signals from the touch sensor increases the stability of the walking motion.

\section{CONCLUSION}

In this paper, the walking motion of a biped robot was analytically examined based on a simple model, and a phase reset of the oscillator

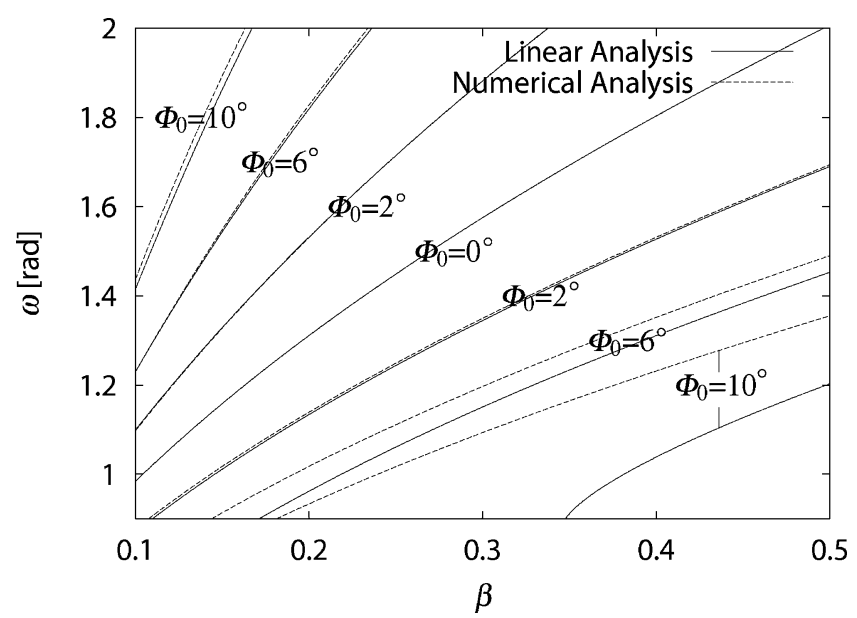

Fig. 9. Optimal value $\Phi_{0}$ with respect to $\beta$ and $\omega$.

that drives the robot was proposed to achieve a robust walking motion. When the swing leg touches the ground, the oscillator receives a feedback signal from the touch sensor at the tip of the leg. Instantly, the phase of the oscillator is reset, and the step period and the walking motion are modified depending on the phase reset. As a result, not only the stability, but also the stability region with respect to the parameters, model size, and walking speed are improved by resetting the phase into an adequate value, i.e., the analytical results demonstrate that the robot can walk adaptively by using the phase reset due to sensory feedbacks.

\section{APPENDIX \\ DERIVATION OF MATRICES $B, D$, AND $E$}

Here, matrices $B, D$, and $E$ are derived based on the linearized equations, conditions, and periodic solutions given above. First, from (10), the following matrix is obtained:

$$
D_{q} h(q)=\left[\begin{array}{cccc}
-1 & 0 & 0 & 0 \\
0 & 1 & 0 & 0 \\
0 & 0 & -\frac{1+c \phi}{1+c \phi_{0}} & \frac{\gamma s \phi}{1+c \phi_{0}} \\
0 & 0 & 0 & 0
\end{array}\right]
$$

By substituting periodic solutions (13) into (26), matrix $B$ is given by

$$
B=\left[\begin{array}{cccc}
-1 & 0 & 0 & 0 \\
0 & 1 & 0 & 0 \\
0 & 0 & -\frac{1-c \phi_{0}}{1+c \phi_{0}} & \frac{S \mathrm{~s} \phi_{0}}{1+c \phi_{0}} \\
0 & 0 & 0 & 0
\end{array}\right]
$$

Second, from (6), it gives

$$
D_{q} r(q)=\left[\begin{array}{llll}
2 & 0 & -(1+c \phi) & -\frac{\dot{\theta}_{2 d}}{\dot{\phi}}
\end{array}\right]^{\mathrm{T}} .
$$


Thus, it follows that

$$
I-\frac{\dot{q} D_{q} r(q)^{\mathrm{T}}}{D_{q} r(q)^{\mathrm{T}} \dot{q}}=\left[\begin{array}{cccc}
\frac{\dot{\theta}_{2 d}}{2 \dot{\theta}_{1}-\dot{\theta}_{2 d}} & 0 & \frac{\dot{\theta}_{1}(1+c \phi)}{2 \dot{\theta}_{1}-\dot{\theta}_{2 d}} & \frac{\frac{\dot{\theta}_{1} \dot{\theta}_{2 d}}{\dot{\phi}}}{2 \dot{\theta}_{1}-\dot{\theta}_{2 d}} \\
\frac{-2 \ddot{\theta}_{1}}{2 \dot{\theta}_{1}-\dot{\theta}_{2 d}} & 1 & \frac{\ddot{\theta}_{1}(1+c \phi)}{2 \dot{\theta}_{1}-\dot{\theta}_{2 d}} & \frac{\frac{\ddot{\theta}_{2 d}}{\dot{\phi}}}{2 \dot{\theta}_{1}-\dot{\theta}_{2 d}} \\
0 & 0 & 1 & 0 \\
\frac{-2 \dot{\phi}}{2 \dot{\theta}_{1}-\dot{\theta}_{2 d}} & 0 & \frac{\dot{\phi}(1+c \phi)}{2 \dot{\theta}_{1}-\dot{\theta}_{2 d}} & \frac{2 \dot{\theta}_{1}}{2 \dot{\theta}_{1}-\dot{\theta}_{2 d}}
\end{array}\right]_{(28)}
$$

The substitution of periodic solutions (13) into (28) gives

$$
D=\left[\begin{array}{cccc}
\frac{-\dot{\theta}_{2 d}^{-}}{2 \dot{\theta}_{1}^{-}-\dot{\theta}_{2 d}^{-}} & 0 & \frac{\dot{\theta}_{1}^{-}\left(1-c \phi_{0}\right)}{2 \dot{\theta}_{1}^{-}-\dot{\theta}_{2 d}^{-}} & \frac{\frac{\dot{\theta}_{1}^{-} \dot{\theta}_{2 d}^{-}}{\omega}}{2 \dot{\theta}_{1}^{-}-\dot{\theta}_{2 d}^{-}} \\
\frac{-2 \ddot{\theta}_{1}^{-}}{2 \dot{\theta}_{1}^{-}-\dot{\theta}_{2 d}^{-}} & 1 & \frac{\ddot{\theta}_{1}^{-}\left(1-c \dot{\theta}_{0}\right)}{2 \dot{\theta}_{1}^{-}-\dot{\theta}_{2 d}^{-}} & \frac{\frac{\dot{\theta}_{2 d}}{2 \dot{\theta}_{1}^{-}-\dot{\theta}_{2 d}^{-}}}{0} \\
\frac{-2 \omega}{2 \dot{\theta}_{1}^{-}-\dot{\theta}_{2 d}^{-}} & 0 & \frac{\omega\left(1-c \phi_{0}\right)}{2 \dot{\theta}_{1}^{-}-\dot{\theta}_{2 d}^{-}} & \frac{2 \dot{\theta}_{1}^{-}}{2 \dot{\theta}_{1}^{-}-\dot{\theta}_{2 d}^{-}}
\end{array}\right] .
$$

Next, from (10), we obtain

$$
D_{q} f(q)=\left[\begin{array}{cccc}
0 & 1 & 0 & 0 \\
1 & 0 & \beta(1+c \phi) & -\beta \gamma \mathrm{s} \phi \\
0 & 0 & 0 & 0 \\
0 & 0 & 0 & 0
\end{array}\right]
$$

Here, perturbation $\hat{q}_{i}(\tau)$ is defined as $\hat{q}_{i}(\tau)=\left[\hat{\theta}_{1 i}(\tau), \dot{\hat{\theta}}_{1 i}(\tau)\right.$, $\left.\hat{\gamma}_{i}(\tau), \hat{\phi}_{i}(\tau)\right]^{T}$. The solutions to (20) with initial conditions $\hat{q}_{i}^{+}=\hat{q}_{i}(0)=\left[\hat{\theta}_{1 i}^{+}, \dot{\hat{\theta}}_{1 i}^{+}, \hat{\gamma}_{i}^{+}, \hat{\phi}_{i}^{+}\right]^{T}$ are obtained by

$$
\begin{aligned}
& \hat{\theta}_{1 i}(\tau)= \frac{e^{\tau}+e^{-\tau}}{2} \hat{\theta}_{1 i}^{+}+\frac{e^{\tau}-e^{-\tau} \dot{\hat{\theta}}_{1 i}^{+}}{2} \\
&+\left\{\left[\frac{e^{\tau}+e^{-\tau}}{2} \mathrm{c} \phi_{0}-\frac{e^{\tau}-e^{-\tau}}{2} \omega \mathrm{s} \phi_{0}\right.\right. \\
&\left.\left.\quad-\mathrm{c}\left(\omega \tau+\phi_{0}\right)\right] \frac{\beta}{\omega^{2}+1}+\beta \frac{e^{\tau}+e^{-\tau}-2}{2}\right\} \hat{\gamma}_{i}^{+} \\
&-\left\{\frac{e^{\tau}+e^{-\tau}}{2} \mathrm{~s} \phi_{0}+\frac{e^{\tau}-e^{-\tau}}{2} \omega \mathrm{c} \phi_{0}\right. \\
&\left.\quad-\mathrm{s}\left(\omega \tau+\phi_{0}\right)\right\} \frac{\beta S}{\omega^{2}+1} \hat{\phi}_{i}^{+}, \quad 0 \leq \tau \leq T \\
& \hat{\gamma}_{i}(\tau)=\hat{\gamma}_{i}^{+}, \quad 0 \leq \tau \leq T \\
& \hat{\phi}_{i}(\tau)=\hat{\phi}_{i}^{+}, \quad 0 \leq \tau \leq T .
\end{aligned}
$$

where

$$
\begin{aligned}
& E_{13}=\frac{\beta}{\omega^{2}+1}\left(\left(E_{+}+1\right) \mathrm{c} \phi_{0}-E_{-} \omega \mathrm{s} \phi_{0}\right)+\beta\left(E_{+}-1\right) \\
& E_{23}=\frac{\beta}{\omega^{2}+1}\left(E_{-} \mathrm{c} \phi_{0}-\left(E_{+}-1\right) \omega \mathrm{s} \phi_{0}\right)+\beta E_{-} \\
& E_{14}=-\frac{\beta S}{\omega^{2}+1}\left(\left(E_{+}-1\right) \mathrm{s} \phi_{0}+E_{-} \omega \mathrm{c} \phi_{0}\right) \\
& E_{24}=-\frac{\beta S}{\omega^{2}+1}\left(E_{-} \mathrm{s} \phi_{0}+\left(E_{+}+1\right) \omega \mathrm{c} \phi_{0}\right) .
\end{aligned}
$$

\section{REFERENCES}

[1] S. Aoi, K. Tsuchiya, and K. Tsujita, "Turning control of a biped locomotion robot using nonlinear oscillators," in Proc. Int. Conf. Robot. Autom., 2004, pp. 3043-3048.

[2] S. Aoi and K. Tsuchiya, "Self-stability of a simple walking model driven by a rhythmic signal," Nonlinear Dyn., to be published.

[3] M. Coleman, A. Chatterjee, and A. Ruina, "Motions of a rimless spoked wheel: A simple three-dimensional system with impacts," Dyn. Stability Syst., vol. 12, no. 3, pp. 139-160, 1997.

[4] Y. Fukuoka, H. Kimura, and A. Cohen, "Adaptive dynamic walking of a quadruped robot on irregular terrain based on biological concepts," Int. J. Robot. Res., vol. 22, no. 3, pp. 187-202, 2003.

[5] A. D. Kuo, "The relative roles of feedforward and feedback in the control of rhythmic movements," Motor Control, vol. 6, no. 2, pp. 129-145, 2002.

[6] S. Grillner, "Neurobiological bases of rhythmic motor acts in vertebrates," Science, vol. 228, pp. 143-149, 1985.

[7] J. W. Grizzle, G. Abba, and F. Plestan, "Asymptotically stable walking for biped robots: Analysis via systems with impulse effects," IEEE Trans. Autom. Control, vol. 46, no. 1, pp. 51-64, Jan. 2001.

[8] A. J. Ijspeert, "A connectionist central pattern generator for the aquatic and terrestrial gaits of a simulated salamander," Biol. Cybern., vol. 84, pp. 331-348, 2001

[9] S. Mochon and T. McMahon, "Ballistic walking: An improved model," Math. Biosci., vol. 52, pp. 241-260, 1980.

[10] J. Nakanishi, J. Morimoto, G. Endo, G. Cheng, S. Schaal, and M. Kawato, "Learning from demonstration and adaptation of biped locomotion," Robot. Auton. Syst., vol. 47, pp. 79-91, 2004.

[11] N. Ogihara and N. Yamazaki, "Generation of human bipedal locomotion by a bio-mimetic neuro-musculo-skeletal model," Biol. Cybern., vol. 84, pp. 1-11, 2001.

[12] G. N. Orlovsky, T. Deliagina, and S. Grillner, Neuronal Control of Locomotion: From Mollusc to Man. Oxford, U.K.: Oxford Univ. Press, 1999.

[13] G. Taga, Y. Yamaguchi, and H. Shimizu, "Self-organized control of bipedal locomotion by neural oscillators," Biol. Cybern., vol. 65, pp. 147-159, 1991.

[14] G. Taga, "A model of the neuro-musculo-skeletal system for human locomotion II.-Real-time adaptability under various constraints," Biol. Cybern., vol. 73, pp. 113-121, 1995.

[15] S. Aoi and K. Tsuchiya, "Locomotion control of a biped robot using nonlinear oscillators," Auton. Robots, vol. 19, no. 3, pp. 219-232, 2005.

[16] K. Tsujita, K. Tsuchiya, and A. Onat, "Adaptive gait pattern control of a quadruped locomotion robot," in Proc. Int. Conf. Intell. Robots Syst., 2001, pp. 2318-2325.

[17] T. Yamasaki, T. Nomura, and S. Sato, "Possible functional roles of phase resetting during walking," Biol. Cybern., vol. 88, pp. 468-496, 2003.

[18] S. Aoi and K. Tsuchiya, "Bifurcation and chaos of a simple walking model driven by a rhythmic signal," Int. J. Non-Linear Mech., vol. 41 , no. 3, pp. 438-446, Mar. 2006.

From (21) and (30), matrix $E$ is given by

$$
E=\left[\begin{array}{cccc}
E_{+} & E_{-} & E_{13} & E_{14} \\
E_{-} & E_{+} & E_{23} & E_{24} \\
0 & 0 & 1 & 0 \\
0 & 0 & 0 & 1
\end{array}\right]
$$

